\title{
CARACTERÍSTICAS BIOLÓGICAS DE TRICHOGRAMMA EXIGUUM EM OVOS DE ANAGASTA KUEHNIELLA E SITOTROGA CEREALELLA
}

\author{
BIOLOGICAL CHARACTERISTICS OF TRICHOGRAMMA EXIGUUM IN THE \\ EGGS OF ANAGASTA KUEHNIELLA END SITOTROGA CEREALELLA
}

\author{
Dirceu Pratissoli ${ }^{1}$; Leandro Pin Dalvi ${ }^{1 *}$; Ricardo Antonio Polanczyk ${ }^{1}$; Gilberto Santos Andrade ${ }^{2}$, \\ Anderson Mathias Holtz ${ }^{1}$; Henrique Otes Nicoline ${ }^{1}$
}

\section{RESUMO}

O objetivo deste trabalho foi obter informações sobre alguns aspectos biológicos de Trichogramma exiguum Pinto \& Platiner, (Hymenoptera: Trichogrammatidae) criado nos hospedeiros alternativos Anagasta kuehniella Zeller (Lepidoptera: Pyralidae) e Sitotroga cerealella Oliver (Lepidoptera: Gelechiidae), visando à produção desses parasitóides para utilização no controle biológico de pragas. A maior taxa de parasitismo de T. exiguum foi encontrada em ovos de A. kuehniella. A viabilidade foi superior em ovos de $S$. cerealella. A razão sexual foi semelhante para ambos os hospedeiros alternativos. Estes resultados demonstram que ambos hospedeiros alternativos podem ser utilizados na criação massal de T. exiguum, porém levando em consideração a importância da taxa de parasitismo, A. kuehniella demonstrou maior aptidão.

Palavras-chave: insecta, controle biológico, criação massal, parasitóides de ovos.

\begin{abstract}
The objective of this work was to evaluate information about biological characteristics of egg of parasitoid Trichogramma exiguum Pinto \& Platiner, (Hymenoptera: Trichogrammatidae) on the alternative hosts Anagasta kuehniella Zeller (Lepidoptera: Pyralidae) and Sitotroga cerealella Oliver (Lepidoptera: Gelechiidae) aiming to use in biological control programs. The higher rate of parasitism of the $\mathrm{T}$. exiguum was verified on eggs of $\mathrm{A}$. kuehniella. The viability of eggs was higher on eggs of $\mathrm{S}$. cerealella. There is not influence in both alternative hosts in the sex ratio. Both alternative hosts can be used in massal rearing of T. exiguum, however due to the importance of the rate of parasitism, A. kuehniella is better adapting.
\end{abstract}

Key words: insecta, biological control, massal rearing, egg parasitoid.

\section{INTRODUÇÃO}

Os parasitóides de ovos do gênero Trichogramma, apresentam ampla distribuição geográfica e grande número de hospedeiros naturais, especialmente os da ordem Lepidoptera, sendo criados massalmente em vários países utilizando-se diversos hospedeiros alternativos (Zucchi \& Monteiro, 1997; Parra, 2002). As mariposas-praga de grãos são preferidas, por serem de fácil criação e multiplicação, baixo custo e por não afetarem o desenvolvimento desse inimigo natural (Navarro, 1998). De acordo com Greenberg et al. (1998), Sitotroga cerealella Olivier (Lepidoptera: Gelechiidae), Anagasta kuehniella Zeller (Lepidoptera: Pyralidae) e Corcyra cephalonica Staiton (Lepidoptera: Pyralidae) são os hospedeiros

* Autor para correspondência (leandro-mpv@cca.ufes.br)

1 Centro de Ciências Agrárias da UFES; Alto Universitário s/nº, C. Postal - 16, CEP: 29500-000 - Alegre - ES; e-mail: dirceu @ npd.ufes.br; leandro-mpv@cca.ufes.br; ricardo@cca.ufes.br; aholtz@insecta.ufv.br; otes.hn@bol.com.br

2 Dept $^{\mathrm{o}}$ Entomologia, Universidade Federal de Viçosa, Viçosa-MG. gilberto.br@ click21.com.br

Fecha de Recepción: 01 Octubre 2007

Fecha de Aceptación: 16 Enero 2008 
alternativos mais utilizados em todo mundo para criação massal destes parasitóides.

Entretanto as características físico-químicas particulares de cada hospedeiro podem afetar tanto a aceitação quanto a adaptação da espécie e/ou linhagem do parasitóide, interferindo nas características biológicas e na sua agressividade, comprometendo assim a qualidade e eficiência do mesmo. Desta forma a escolha do hospedeiro alternativo mais adequado é o primeiro passo no processo de produção massal de parasitóides do gênero Trichogramma (Monge et al.,1999).

No passado, muitos insucessos na utilização de parasitóides ocorreram pela falta de conhecimentos básicos durante a criação massal, como as interações entre parasitóide e hospedeiro. Sendo assim, objetivou-se com este trabalho avaliar algumas características de Trichogramma exiguum Pinto \& Platiner, (Hymenoptera: Trichogrammatidae) criado sobre ovos dos hospedeiros alternativos A. kuehniella e $S$. cerealella, visando à produção destes parasitóides para utilização no controle biológico de pragas.

\section{MATERIAL E MÉTODOS}

O experimento foi conduzido no Núcleo de Desenvolvimento Científico e Tecnológico em Manejo Fitossanitário "NUDEMAFI" situado no campus do Centro de Ciências Agrárias da UFES (CCA-UFES), Alegre-ES, em câmara climatizada com temperatura de $25 \pm 1^{\circ} \mathrm{C}$, umidade relativa de $70 \pm 10 \%$ e fotofase de 14 horas.

A linhagem de T. exiguum utilizada foi coletada com armadilhas contendo ovos de A. kuehniella, em plantios comerciais de tomate no município de Piaçú, Espírito Santo, sendo os espécimes obtidos, divididos em dois lotes, um criado em ovos de A. kuehniella e o outro em ovos de $S$. cerealella. Ambos os lotes foram mantidos em laboratório por 10 gerações até o inicio dos experimentos.

A metodologia empregada na criação do hospedeiro alternativo A. kuehniella foi à desenvolvida por Parra et al. (1997), porém foi adaptada para as condições do setor Entomologia do NUDEMAFI, utilizando uma dieta à base de farinha de trigo integral e de milho (97\%) e levedura de cerveja (3\%).

O preparo da dieta para a criação do hospedeiro $S$. cerealella teve como base à metodologia desenvolvida por Hassan (1997), com algumas modificações, utilizando uma dieta à base de grãos de trigo descarificado, com umidade variável entre 11-14\%. As condições climáticas da sala de criação de A. kuehniella e $S$. cerealella foram de $25 \pm 2{ }^{\circ} \mathrm{C}$ e umidade relativa $70 \pm 10 \%$.

A criação e multiplicação do parasitóide T. exiguum baseou-se na metodologia descrita por Parra et al. (1997). Ovos de $S$. cerealella e A. kuehniella colados com goma arábica diluída a $10 \%$ em retângulos de cartolina azul celeste (cartelas) $(4,0 \times 2,0 \mathrm{~cm})$, foram oferecidos aos parasitóides em recipientes de vidro $(8,5 \times 2,4 \mathrm{~cm})$, onde foi permitido o parasitismo por 24 horas. Os ovos de A. kuehniella foram inviabilizados em lâmpada germicida por 50 minutos, devido a esta espécie apresentar habito canibal.

Para cada hospedeiro avaliado foram utilizadas 25 fêmeas recém emergidas individualizadas em tubos de vidro $(4,0 \times 0,7 \mathrm{~cm})$, contendo gotículas de mel de abelha para alimentação e tamponados com filme plástico PVC. Cada fêmea constituiu uma repetição, e recebeu 40 ovos do respectivo hospedeiro, colados com goma arábica diluída a $10 \%$ em um retângulo de cartolina $(3,5 \times 0,5 \mathrm{~cm})$ de cor azul-celeste. Foi permitido o parasitismo por 24 horas, sendo que ao término a cartela era trocada por uma nova. O procedimento foi realizado durante três dias devido a este período responder normalmente por cerca de $70 \%$ do parasitismo total. As cartelas retiradas a cada 24 horas foram acondicionadas em tubos de vidro $(8,5 \times 2,4 \mathrm{~cm})$ e mantidas nas mesmas condições de criação do parasitóide até a emergência dos descendentes.

Foram avaliadas a percentagem de parasitismo, a viabilidade dos ovos e a razão sexual de $T$. exiguum criado nos hospedeiros alternativos A. kuehniella $\mathrm{e}$ $S$. cerealella. O delineamento utilizado foi o inteiramente casualizado, as médias foram comparadas pelo teste de Tukey a 5\%.

\section{RESULTADOS E DISCUSSÃO}

Ambos hospedeiros alternativos foram aceitos por T. exiguum. No entanto, foi observado que a taxa parasitismo foi maior em ovos de A. kuehniella nos três dias de avaliação em que foram oferecidas cartelas contendo ovos do hospedeiro (Tabela 1). Comportamento semelhante a este foi encontrado por Gomes (1997), quando avaliou a agressividade de cinco linhagens $T$. pretiosum, encontrando também 
Tabela 1

Valores médios \pm EP de taxa de parasitismo, viabilidade e razão sexual de Trichogramma exiguum, em ovos de Anagasta kuehniella e Sitotroga cerealella. $\left(25 \pm 1^{\circ} \mathrm{C}\right.$, $\mathrm{UR}=70 \pm 10 \%$ e fotofase $=14 \mathrm{~h})$. Alegre, UFES, 2006

\begin{tabular}{|l|c|c|c|}
\hline \multirow{2}{*}{ Hospedeiro } & \multicolumn{3}{|c|}{ Idade do parasitóide } \\
\cline { 2 - 4 } & 1 dia & 2 dias & 3 dias \\
\hline & \multicolumn{3}{|c|}{ Taxa de parasitismo $(\%)$} \\
\hline A. kuehniella & $36,65 \pm 1,41^{\mathrm{a}}$ & $17,36 \pm 0,95^{\mathrm{a}}$ & $19,21 \pm 2,09^{\mathrm{a}}$ \\
\hline S. cerealella & $27,08 \pm 1,12^{\mathrm{b}}$ & $12,70 \pm 2,30^{\mathrm{b}}$ & $9,68 \pm 2,38^{\mathrm{b}}$ \\
\hline & \multicolumn{3}{|c|}{ Viabilidade $(\%)$} \\
\hline A. kuehniella & $90,58 \pm 1,52^{\mathrm{a}}$ & $86,47 \pm 2,93^{\mathrm{a}}$ & $87,46 \pm 1,23^{\mathrm{a}}$ \\
\hline S. cerealella & $93,70 \pm 2,96^{\mathrm{a}}$ & $90,97 \pm 1,97^{\mathrm{a}}$ & $91,21 \pm 2,41^{\mathrm{a}}$ \\
\hline A. kuehniella & $1,00 \pm 0,0^{\mathrm{a}}$ & $1,00 \pm 0,0^{\mathrm{a}}$ & $0,96 \pm 0,02^{\mathrm{a}}$ \\
\hline S. cerealella & $1,00 \pm 0,0^{\mathrm{a}}$ & $1,00 \pm 0,0^{\mathrm{a}}$ & $1,00 \pm 0,00^{\mathrm{a}}$ \\
\hline
\end{tabular}

Pares de médias seguidas pela mesma letra minúscula na coluna não diferem entre si pelo teste de Tukey a $\mathrm{p} \leq 0,05$.

menor aceitação em ovos de $S$. cerealella. Vários são os fatores que podem afetar o parasitismo de Trichogramma, como por exemplo, o tamanho do ovo, espessura do córion e a presença de químicos voláteis. Contudo, no caso de A. kuehniella e $S$. cerealella a qualidade nutricional é o fator que mais influencia na aceitação do parasitóide, sendo os ovos de A. kuehniella mais nutritivos em relação aos ovos de S. cerealella (Pratissoli \& Parra, 2000).

Tanto em ovos de S. cerealella, quanto em ovos de A. kuehniella, a viabilidade do parasitóide foi superior a $86 \%$ (Tabela 1). Esta viabilidade é considerada satisfatória no manual de controle de qualidade de produção de Trichogramma (Navarro, 1998). Resultados encontrados por Alencar et al.

\section{LITERATURA CITADA}

ALENCAR, J.A.; HAJI, F.N.P.; OLIVEIRA, J.V.; MOREIRA, A.N. 2000. Biologia de Trichogramma pretiosum Riley em ovos de Sitotroga cerealella (Olivier). Pesquisa Agropecuária Brasileira. 35: 1669-1674.

GOMES, S.M. 1997. Comparação de três hospedeiros alternativos para criação e produção massal de Trichogramma pretiosum e T. galloi. Piracicaba, ESALQ, 106 p. (Disertação de mestrado).

GONÇALVES, J.R.; HOLTZ, A.M.; PRATISSOLI, D.; GUEDES, R.N.C. 2003. Avaliação da qualidade de
(2000) atestam a adequação de $S$. cerealella para T. pretiosum com valor de $88 \%$ de viabilidade. Nicoli et al., (2004) estudando a viabilidade de $T$. atopovirilia em ovos de A. kuehniella obteve $96 \%$ nestas mesmas condições, confirmando a qualidade do hospedeiro.

A razão sexual dos descendentes de T. exiguum criados em ovos de A. kuehniella foi semelhante a $S$. cerealella (Tabela 1), demonstrando que com a utilização destes hospedeiros pode-se produzir um alto número de fêmeas do parasitóide e atingir o índice aceitável, exigido no controle de qualidade de Trichogramma, com valor igual ou superior a 0,5 (Navarro, 1998). Gonçalves et al. (2003) demonstraram que o valor de razão sexual para $T$. pretiosum não foi afetado, ficando também acima de 0,5. De acordo com Vinson, (1994), o sexo da progênie pode ser influenciado pela qualidade do hospedeiro demonstrado que de acordo com os resultados ambos hospedeiros se mostram adequados para o desenvolvimento desta espécie de parasitóide.

\section{CONCLUSÃO}

Ambos hospedeiros alternativos podem ser utilizados na criação massal de T. exiguum, porém, levando em consideração a importância da taxa de parasitismo, A. kuehniella demonstrou maior aptidão.

\section{AGRADECIMENTOS}

Ao Conselho Nacional de Desenvolvimento Cientifico e Tecnológico (CNPq) e à Coordenação de Aperfeiçoamento de Pessoal de Nível Superior (CAPES) pela concessão de bolsas.
Trichogramma pretiosum (Hymenoptera: Trichogrammatidae) em ovos de Sitotroga cerealella (Lepidoptera: Gelechiidae). Acta Scientiarum. Agronomy. 25: 485-489.

GREENBERG, S.M.; MORRISON, R.K.; NORDLUND, O.A.; KING, E.G. 1998. A review of thescientitic literature and methods for production of factitions hosts for use in mass rearing of Trichograrnma (Hym.: Trichogrammatidae) in the former Soviet Union, the United States, Western Europe and China. Journal Entomology Science. 33: 15-32. 
HASSAN, S.A. 1997. Criação da traça do milho Sitotroga cerealella, para produção massal de Trichogramma. In: PARRA, J.R.P.; ZUCCHI, R.A., Trichogramma e o controle biológico. Piracicaba: FEALQ. 324 p.

MONJE, J.C.; ZEBITZ, C.P.W.; OHNESORGE, B. 1999. Host and host age preference of Trichogramma galloi and T. pretiosum (Hym.:Trichogrammatidae) reared on different hosts. Journal Economical of Entomolology. 92: 97-103.

NAVARRO, M.A. 1998. Trichogramma spp. Procucción, Uso y Manejo en Colombia. Guadalajara de Buga: Impretec. 176 p.

NICOLI, E.M.; PRATISSOLI, D.; REIS, E.F.; SANTOS, H.S 2004. Viabilidade e razão sexual de Trichogramma atopovirilia Oatman \& Platner, 1983 (Hymenoptera, Trichogrammatidae) sob influência do hospedeiro Anagasta kuehniella (Zeller, 1879) (Lepidoptera, Pyralidae) em condições de laboratório. Entomologia y Vecterinaria. 11: 521-533.

PARRA, J.R.P. 1997. Técnicas de criação de Anagasta kuehniella, hospedeiro alternativo para produção de Trichogramma. In:
PARRA, J.R.P.; ZUCCHI, R. A. Trichogramma e o controle biológico aplicado. Piracicaba: FEALQ. 324 p.

PARRA, J.R.P. 2002. Criação massal de inimigos naturais. In: PARRA, J.R.P., BOTELHO, P.S.M., CORRÊA-FERREIRA, BENTO, J.M.S. Controle biológico no Brasil: Parasitóides e Predadores. Barueri, Manole. 609 p.

PRATISSOLI, D.; PARRA, J.R.P. 2000. Desenvolvimento e exigências térmicas de Trichogramma pretiosum Riley, criados em duas traças do tomateiro. Pesquisa Agropecuária Brasileira. 35: 1281-1288.

VINSON, S.B. 1994. Physiological interactions between egg parasitoids and their hosts. In: WAJNBERG, E.; HASSAN, S A. (eds.) Biological control with egg parasitoids. Wallingford: CAB International. 286 p.

ZUCCHI, R.A.; MONTEIRO, R.C. 1997. O gênero Trichogramma na América do Sul. In: PARRA, J. R. P.; ZUCCHI, R. A. (eds.), Trichogramma e o Controle Biológico Aplicado, FEALQ. 324 p 